\title{
Schmerzmessung durch Selbstbeurteilung bei älteren Menschen mit kognitiver Beeinträchtigung
}

\section{Pain Assessment in Older Adults with Cognitive Impairment}

Autoren

Institut
A. M. Aegerter, J. Kool

Institut für Physiotherapie, Dept. Gesundheit, Zürcher Hochschule für Angewandte Wissenschaften (ZHAW), CH-Winterthur
Schlüsselwörter

- Schmerzmessung

- kognitive Beeinträchtigung

- Demenz

\section{Key words}

- pain measurement

- cognitive impairment

- dementia eingereicht 17.3.2015

akzeptiert $\quad 27.10 .2015$

\section{Bibliografie}

DOI http://dx.doi.org/ 10.1055/s-0035-1567062 physioscience 2016; 12: 26-29 (c) Georg Thieme Verlag KG Stuttgart · New York . ISSN 1860-3092

\section{Korrespondenzadresse Andrea Martina Aegerter, BSC PT, MSc PT (in Ausbildung) Zürcher Hochschule für Angewandte Wissenschaften (ZHAW), Institut für Physiotherapie, Dept. Gesundheit Technikumstr. 71 8401 Winterthur Schweiz andrea.aegi@hotmail.com}

\section{Zusammenfassung}

$\nabla$

Hintergrund: Bei älteren Personen mit kognitiver Beeinträchtigung werden Schmerzen oft unteroder überversorgt. Die Basis einer Schmerztherapie stellt die Schmerzerfassung dar.

Ziel: Die Arbeit erforschte die optimalste Selbstbeurteilungsskala zur Schmerzerfassung bei älteren Personen mit einer kognitiven Beeinträchtigung bezüglich der Kriterien der Anwendbarkeit, Konstruktvalidität und Retest-Reliabilität.

Methode: Verschiedene Datenbanken wurden nach Studien durchsucht, die Selbstbeurteilungsskalen zur Schmerzerfassung verwendeten. Die verschiedenen Skalen wurden hinsichtlich Anwendbarkeit, Konstruktvalidität und Retest-Reliabilität miteinander verglichen.

Ergebnisse: Vier Studien erfüllten alle Auswahlkriterien. Bei Personen mit leichter kognitiver Beeinträchtigung schnitt die verbale Beurteilungsskala mit einer Anwendbarkeit von über $90 \%$ am besten ab (Mini-Mental-Status-Test: 18-23 Punkte). Die Korrelation der Ergebnisse unterschiedlicher Skalen wies auf valide Messungen hin. Die Retest-Reliabilität war nur bei einer Intrarater-Messung genügend. Bei einer mäßigen bis starken kognitiven Beeinträchtigung war die Anwendbarkeit bei allen Skalen zu gering (<90\%). Schlussfolgerung: Bei einer leichten kognitiven Beeinträchtigung stellt die verbale Beurteilungsskala ein geeignetes Messinstrument dar. Bei älteren Personen mit einer stärkeren kognitiven Beeinträchtigung sind Schmerzskalen zur Selbstbeurteilung nicht einsetzbar.

\section{Einleitung}

Bei älteren Menschen sind Schmerzen ein häufiges Problem. Helme und Gibson [9] zufolge betrifft dies bis zu 80\% der Bewohner von Pflegeinstitutionen. Schmerzen bei älteren, an Demenz erkrank-

\section{Abstract \\ v}

Background: In elderly people with cognitive impairment pain is often under- or overtreated. Pain assessment is considered to be the basis of pain management.

Objective: This article investigated the most suitable self-assessment scale regarding applicability, construct validity and retest reliability for pain assessment in elderly people with cognitive impairment.

Method: Various databases were searched for studies using self-assessment scales for pain assessment. The different scales were compared regarding applicability, construct validity and retest reliability.

Results: Four studies met all selection criteria. In people with mild cognitive impairments the verbal rating scale performed best with more than 90\% (mini-mental state examination: 18-23 points). The correlation of the different scales' results implied valid measurements. Retest-reliability was only sufficient in an intrarater-testing. The applicability for moderate to severe cognitive impairment was too low in all the scales $(<90 \%)$. Conclusion: For mild cognitive impairment the verbal rating scale is considered to be a suitable assessment tool. In elderly people with more severe cognitive impairments pain assessment scales are not useful for self-assessment. ten Personen bleiben oft unterversorgt [15]. Im klinischen Alltag lässt sich zugleich beobachten, dass eine Äußerung, z. B. ein Schrei aus Angst oder Verwirrung als Schmerz fehlinterpretiert und ein Schmerzmittel verabreicht wird, was zu einer Überversorgung führen kann. 
Das Fundament einer gezielten Schmerztherapie bilden die Schmerzerkennung und -erfassung [23]. Letztere kann bei älteren Menschen mit kognitiver Beeinträchtigung deutlich erschwert sein $[2,25]$. Laut Stolee et al. [24] gilt es, bei diesen Personen nach einem Schmerzerfassungsinstrument zu suchen, da kein von ihnen untersuchtes Instrument genügend reliabel und valide war und somit empfohlen werden konnte. Dies erscheint mit Blick auf den demografischen Wandel und die stetig älter werdende Bevölkerung umso wichtiger.

Eine aussagekräftige Schmerzanamnese ist maßgeblich für den Erfolg der Behandlung verantwortlich. Das Ziel dieses Reviews war es zu untersuchen, welche Skala bezüglich der Anwendbarkeit, Konstruktvalidität und Retest-Reliabilität zur Schmerzerfassung bei älteren Personen mit kognitiver Beeinträchtigung am besten geeignet ist und inwiefern das Ausmaß der kognitiven Beeinträchtigung die Anwendung einer Selbstbeurteilung erlaubt. Da Selbstbeurteilung eine weit verbreitete und praktikable Methode darstellt und den Schmerz als subjektive Empfindung berücksichtigt, wurden hierzu ausschließlich selbstbeurteilende Schmerzskalen berücksichtigt.

\section{Methode}

\section{Literatursuche}

In den Datenbanken PubMed und CINAHL erfolgte im Januar 2013 die Suche nach wissenschaftlichen Studien. Für die gezielte Recherche dienten die mit den Boole-Operatoren „AND“ und „OR“ verknüpften Schlagwörter „pain measurement“, „cognition disorders“ und „dementia“. Zur Eingrenzung der Ergebnisse kamen 3 Filter zum Einsatz (Alter $65+$, englisch- und deutschsprachige Publikationen aus den letzten 10 Jahren). Die weitere Selektierung der Publikationen geschah anhand des Titels und Abstracts sowie unter Verwendung folgender Einschlusskriterien: Vergleich der verbalen Beurteilungsskala mit anderen Schmerzskalen, Gruppeneinteilung anhand der kognitiven Beeinträchtigung (Mini-MentalStatus-Test), prozentuale Anwendbarkeit (für jede kognitive Gruppe einzeln berechnet) und die Ermittlung der Konstruktvalidität und/oder Retest-Reliabilität der Schmerzskalen.

Die Studien untersuchten die Konstruktvalidität, indem sie unterschiedliche Schmerzskalen verglichen. Da ein Referenztest für die Schmerzmessung nicht verfügbar ist, lässt sich die Kriteriumsvalidität nicht beurteilen. Weiter fand ein Vergleich der Anwendbarkeit der verbalen Beurteilungsskala mit der anderer Skalen statt. Vom Skaleneinsatz wurden die Konstruktvalidität (Rangkorrelationskoeffizient nach Spearman) und die Retest-Reliabilität (Intraklassen-Korrelationskoeffizient) ermittelt. Bei den Gesamtergebnissen fand das Risiko für Bias und Verzerrung der Ergebnisse in den einzelnen Studien Berücksichtigung. Dieses Risiko nimmt bei geringer methodologischer Qualität zu.

\section{Definition und Instrumente}

Die Anwendbarkeit ist der prozentuale Anteil der Patienten, bei welchen die Skala erfolgreich eingesetzt werden kann. Dies lässt sich beispielsweise überprüfen, indem die Patienten die Skalenanwendung erklären müssen.

Für den Vergleich der ausgewählten Studien, die Gewichtung der Resultate und die Beurteilung des Risikos für Bias erfolgte die Analyse mit der Quality Appraisal for Reliability Studies Checklist (QAREL; [14]). Dieses besteht aus einem Fragebogen mit 11 Items und erlaubt eine Aussage über die diagnostische Reliabilität einer Studie.
Eine Erfassungsmöglichkeit für kognitive Defizite bietet der MiniMental-Status-Test (MMST, [6], dessen Ergebnis von bestmöglichen 30 (uneingeschränkt) bis zu 0 Punkten (schwerstmögliche Beeinträchtigung) reicht (30 - 24 Punkte: keine kognitive Beeinträchtigung; 23 - 18 Punkte: leichte kognitive Beeinträchtigung; 17 - 10 Punkte: mäßige kognitive Beeinträchtigung; 9 - 0 Punkte: starke kognitive Beeinträchtigung; [3]).

\section{Ergebnisse}

\section{Literatursuche}

Die Literatursuche ergab 516 Publikationen. In diesen Review wurden 4 Studien eingeschlossen, die die Verbal Rating Scale (VRS [12]) mit anderen Schmerzskalen verglichen: Color Analogue Scale (CAS [22]), Faces Pain Scale (FPS [11]), Numeric Rating Scale (NRS [12]), Red Wedge Scale (RWS [20]), Visual Analogue Scale (VAS) mit den Varianten vertikale (VVAS) oder horizontale (HVAS) Ausrichtung und mechanisch/mit verschiebbarem Element (MVAS [12]).

Closs et al. [3] verglichen 5 Selbstbeurteilungsskalen bei Personen im Pflegeheim mit unterschiedlicher kognitiver Beeinträchtigung. Die 1. Studie von Pautex et al. [18] untersuchte die Anwendbarkeit und Retest-Reliabilität von 4 Selbstbeurteilungsskalen sowie die Korrelation mit Fremdbeurteilungsskalen bei hospitalisierten älteren und dementen Patienten. Die 2. Studie von Pautex et al. [19] verfolgte die Frage, ob bei starker Demenz eine Selbst- oder Fremdbeurteilung durchgeführt werden sollte. Hierzu vergliche sie die Anwendbarkeit von Selbstbeurteilungsskalen bei stark dementen hospitalisierten Patienten mit Daten aus der Fremdbeurteilung. Personen et al. [20] untersuchten die Anwendbarkeit von 4 Schmerzskalen bei dementen Patienten.

\section{Risiko für Bias}

Die Ein- und Ausschlusskriterien waren in jeder Studie so gewählt, dass sie nicht die ganze Population der älteren Personen mit einer kognitiven Beeinträchtigung berücksichtigten. Die Stichproben sind somit nicht für alle Personen mit einer kognitiven Beeinträchtigung repräsentativ. Dies erschwert eine Aussage über die Konstruktvalidität und die Retest-Reliabilität. Bei den Studien von Closs et al. [3] und Personen et al. [20] fehlten einige Angaben, was einerseits die Praxisempfehlung schwierig gestaltet und andererseits die erhaltenen Resultate infrage stellt. Insgesamt führte die methodologische Beurteilung der Studien mithilfe der QUAREL zu einer höheren Gewichtung der beiden Studien von Pautex et al. $[18,19]$. Alle Studien wiesen jedoch ein hohes Risiko für Bias auf.

\section{Anwendbarkeit}

Von den untersuchten Skalen erreichte bei Patienten mit einer kognitiven Beeinträchtigung keine eine 100\%ige Anwendbarkeit ( Tab. 1). In allen Studien nahm die Anwendbarkeit der untersuchten Skalen mit zunehmender kognitiver Beeinträchtigung ab (Ausnahme: verbale Beurteilungsskala bei Personen et al. [20]). Grundsätzlich schnitt die Befragung mit der VRS besser $a b$ als mit der NRS oder der schriftlichen VAS. Bei leichter kognitiver Beeinträchtigung erzielte die VRS in 2 von 3 Studien eine Anwendbarkeit von über $90 \%$ (100\% [3]; $91 \%$ [18]; 85\% [20]; Durchschnitt: $92 \%$ ) und schnitt somit am besten ab. Bei mäßiger bis starker kognitiver Beeinträchtigung konnte in keiner der Studien ein Instrument eine Anwendbarkeit von über 90\% erreichen. Einzige Ausnahme bildete die VRS bei Closs et al. [3], die je- 
Tab. 1 Anwendbarkeit unterschiedlicher Schmerzskalen bei älteren Menschen in Abhängigkeit der kognitiven Beeinträchtigung.

\begin{tabular}{|c|c|c|c|c|c|}
\hline \multirow[t]{2}{*}{ Studie } & \multirow[t]{2}{*}{ Skala } & \multicolumn{4}{|c|}{ Anwendbarkeit (\%) } \\
\hline & & keine & leicht & mäßig & stark \\
\hline \multirow[t]{5}{*}{ Closs et al. [3] } & VRS & 100 & 100 & 97 & 36 \\
\hline & NRS & 100 & 92 & 87 & 25 \\
\hline & FPS & 91 & 71 & 68 & 25 \\
\hline & CAS & 86 & 88 & 74 & 21 \\
\hline & MVAS & 96 & 79 & 55 & 7 \\
\hline \multirow[t]{4}{*}{ Pautex et al. [18] } & VRS & - & 91 & 73 & 33 \\
\hline & HVAS & - & 97 & 79 & 27 \\
\hline & VVAS & - & 92 & 74 & 27 \\
\hline & FPS & - & 89 & 65 & 27 \\
\hline \multirow[t]{3}{*}{ Pautex et al. [19] } & VRS & - & - & - & 39 \\
\hline & HVAS & - & - & - & 29 \\
\hline & FPS & - & - & - & 49 \\
\hline \multirow[t]{4}{*}{ Pesonen et al. [20] } & VRS & 83 & 85 & 79 & 63 \\
\hline & VAS & 78 & 60 & 57 & 20 \\
\hline & RWS & 83 & 68 & 61 & 22 \\
\hline & FPS & 83 & 68 & 64 & 29 \\
\hline
\end{tabular}

CAS = Color Analogue Scale; FPS = Faces Pain Scale; HVAS = horizontale VAS MVAS = mechanische VAS; NRS = Numeric Rating Scale; RWS = Red Wedge Scale; VAS = Visual Analogue Scale; VRS = Verbal Rating Scale; VVAS= vertikale VAS

doch in den anderen 3 Studien ungenügende Ergebnisse erzielte und somit als inkonsistentes Ergebnis zählt. Bei allen anderen Instrumenten (CAS, FPS, NRS, RWS, horizontale/vertikale/mechanische VAS) war die Anwendbarkeit für die allgemeine Verwendung zu gering.

\section{Konstruktvalidität}

Zur Ermittlung der Konstruktvalidität wurden die Ergebnisse aller verwendeten Schmerzskalen verglichen und auf Zusammenhänge überprüft. Die Studien unterschieden hierbei nicht nach der kognitiven Beeinträchtigung. Diese Korrelation wurde mit dem Rangkorrelationskoeffizient nach Spearman ( $\mathrm{r}$ ) angegeben ( Tab.2). Da alle Messungen mit den Skalen die Schmerzerfassung als Ziel anstreben, sollte ihre Korrelation möglichst hoch sein $(r>0,8)$. Bei Pautex et al. $[18,19]$ waren die Messungen mit Skalen bei Personen bis und mit mäßiger kognitiver Beeinträchtigung valide $(r=0,81$ 0,95). Bei starker kognitiver Beeinträchtigung war die Spannweite der Koeffizienten für eine konkrete Aussage zu groß $(r=0,45$ 0,94). Bei Closs et al. [3] und Pesonen et al. [20] korrelierten die Ergebnisse weniger stark $(r=0,55-0,79$ bzw. $r=0,53-0,72)$, was die Konstruktvalidität der Skalen infrage stellt.

\section{Retest-Reliabilität}

Die beiden Studien von Pautex et al. $[18,19]$ untersuchten anhand der vertikalen/horizontalen VAS, VRS und FPS, ob eine Wiederholung der Messung das gleiche Resultat liefert (Retest-Reliabilität). Dabei nahm einerseits die gleiche Person (Intrarater-Messung) und andererseits 2 verschiedene Personen (Interrater-Messung) die beiden Messungen vor. Die Korrelation wurde mit dem Intraklassen-Korrelationskoeffizienten (ICC) berechnet $($ Tab.3). Je näher der Wert bei 1 liegt, umso höher sind die Korrelation und die Retest-Reliabilität des Instruments. Bei einer Intrarater-Messung weist die hohe Korrelation (ICC >0,93) auf reliable Messungen hin, während die Interrater-Messung mit geringeren Werten (ICC $=0,71-0,9$ bzw. ICC $=0,88-0,95$ ) die Retest-Reliabilität infrage stellt $[18,19]$.
Tab. 2 Konstruktvalidität.

\begin{tabular}{|llll}
\hline Closs et al. [3] & $\begin{array}{l}\text { Pautex et al. } \\
\text { [18] }\end{array}$ & Pautex et al. [19] & Pesonen et al. [20] \\
\hline $\mathrm{r}=0,53-0,72$ & $\mathrm{r}=0,81-0,95$ & $\mathrm{r}=0,45-0,94$ & $\mathrm{r}=0,55-0,79$ \\
\hline $\mathrm{p}=0,000$ & $\mathrm{p}<0,001$ & $\mathrm{p}<0,001$ & $\mathrm{P}<0,001$
\end{tabular}

$\mathrm{p}=$ Signifikanzwert; $r$ = Spearman-Rangkorrelationskoeffizient.

Tab. 3 Retest-Reliabilität.

\begin{tabular}{|lll|}
\hline & Pautex et al. [18] & Pautex et al. [19] \\
\hline Interrater-Messung & ICC $=0,94-0,97$ & ICC $=0,93-0,98$ \\
\hline Intrarater-Messung & ICC $=0,71-0,90$ & ICC $=0,88-0,95$
\end{tabular}

ICC = Intraklassen-Korrelationskoeffizient; Interrater-Messung = 2 Messpersonen; Intrarater-Messung= 1 Messperson.

\section{Diskussion}

\section{$\nabla$}

\section{Hauptergebnis}

Alle eingeschlossenen Studien hatten ein hohes Risiko für Bias. Das bessere Abschneiden der beiden Studien von Pautex et al. $[18,19]$ führte dazu, dass sie höher gewichtet wurden. Bei älteren Personen mit einer leichten kognitiven Beeinträchtigung beträgt die Anwendbarkeit der VRS über 90\%, was als „gut“ gilt. Somit ist die VRS bei der Schmerzerfassung bei älteren Personen mit einer leichten kognitiven Beeinträchtigung allen anderen selbstbeurteilenden Schmerzskalen überlegen. Deshalb ist bei der genannten Gruppe der Einsatz der VRS zu empfehlen. Bei einer mäßigen sowie starken kognitiven Beeinträchtigung ist die Anwendbarkeit für einen sinnvollen Skaleneinsatz ungenügend (<90\%). Die hohe Korrelation der Messergebnisse weist bei Personen bis und mit mäßiger kognitiver Beeinträchtigung auf valide Skalen hin. Die Retest-Reliabilität scheint nur gegeben, wenn die gleiche Person die Messung durchführt.

\section{Vergleich mit anderer Literatur}

Die Ergebnisse stimmen mit denjenigen anderer Studien überein $[5,10,16]$. Sie alle kamen zu dem Schluss, dass bei Personen mit leichter bis mäßiger kognitiver Beeinträchtigung eine Messung mittels Schmerzskala möglich ist. Für die konkrete Empfehlung eines Skaleneinsatzes bedarf es weiterer Forschung auf diesem Gebiet.

Da stark beeinträchtigte Personen keine Selbsterfassung durchführen können, befürworten die Autoren in dieser Gruppe den Einsatz von Fremdbeurteilungen [5, 10, 16]. Ein konkretes Instrument können sie nicht empfehlen.

Ähnliche Empfehlungen für die Schmerzerfassung bei Personen mit kognitiver Beeinträchtigung geben das Royal College of Physicians, die British Geriatrics Society und die British Pain Society [21] sowie Hadjistavropoulos et al. [8]. Zur Fremdbeurteilung nennen sie die in $\downarrow$ Tab. 4 beschriebenen Instrumente.

\section{Theorie-Praxis-Transfer}

Bei Personen mit leichter kognitiver Beeinträchtigung stellt die VRS ein geeignetes Instrument zur Schmerzerfassung dar. Die wissenschaftliche Literatur zeigt, dass der Skaleneinsatz bei stärkerer kognitiver Beeinträchtigung eine zu geringe Anwendbarkeit aufweist und in der Therapie nicht erfolgreich eingesetzt werden kann $[5,10,16]$. Bei diesen Patienten sind Fremdbeurteilungen indiziert. Die systematische Fremdbeurteilung erfasst 
Tab. 4 Fremdbeurteilungsinstrumente.

\begin{tabular}{|c|c|}
\hline Instrument & Beurteilungskriterien \\
\hline $\begin{array}{l}\text { The Pain Assessment } \\
\text { Checklist for Seniors } \\
\text { with Limited Ability } \\
\text { to Communicate } \\
\text { (PACSLAC; [7]) }\end{array}$ & $\begin{array}{l}\text { Mimik, Aktivität, Körperbewegung, Per- } \\
\text { sönlichkeit, Stimmung, soziales/verbales } \\
\text { Verhalten, Änderungen in der Physiologie, } \\
\text { im Essen oder im Schlafen }\end{array}$ \\
\hline Doloplus-2 Scale [13] & $\begin{array}{l}\text { somatische Schmerzreaktionen (z. B. ver- } \\
\text { bale Äußerungen, Mimik), psychomotori- } \\
\text { sche (z. B. Mobilität) sowie psychosoziale } \\
\text { Reaktionen (z. B. Kommunikation) }\end{array}$ \\
\hline Abbey Pain Scale [1] & $\begin{array}{l}\text { verbale Äußerungen (z. B. Wimmern), } \\
\text { Mimik, Änderungen der Körpersprache, des } \\
\text { Verhaltens, der Physiologie oder der Physis }\end{array}$ \\
\hline
\end{tabular}

Verhaltenshinweise wie die Mimik und Bewegung ( $\bullet$ Tab.4). Die vorliegende Arbeit soll auf die Problematik der Unter- und Überversorgung von Schmerzen bei älteren Menschen aufmerksam machen und die Berufsgruppe der Physiotherapeuten für die systematische Schmerzerfassung bei Menschen mit kognitiver Beeinträchtigung sensibilisieren.

\section{Limitationen der Arbeit}

Nicht alle Studien beschrieben genau das Testvorgehen und die Ermittlung des Skalenverständnisses. Deshalb kann die Anwendbarkeit verfälscht und noch höher als berichtet sein. Weiter wurden bei der Anwendbarkeit kein Messfehler angegeben und eine geringe Stichprobengröße gewählt. Da die Konstruktvalidität und Retest-Reliabilität nicht für jede kognitive Gruppe und jede Skala einzeln erhoben wurden, lässt sich keine differenzierte Aussage treffen.

\section{Schlussfolgerungen}

Bei einer leichten kognitiven Beeinträchtigung ist die Verbal Rating Scale (VRS) im Vergleich mit 5 anderen Schmerzerfassungsskalen (Color Analogue Scale, Faces Pain Scale, Numeric Rating Scale, Red Wedge Scale, Visual Analogue Scale) das geeignetste Instrument zur Selbstbeurteilung von Schmerzen. Bei älteren Personen mit mäßiger oder starker kognitiver Beeinträchtigung können selbstbeurteilende Schmerzskalen nicht eingesetzt werden.

\section{Ouintessenz}

Zur Vermeidung einer Unter- und Überversorgung in der Schmerzbehandlung von Patienten mit einer leichten kognitiven Beeinträchtigung stellt die Verbal Rating Scale (VRS) ein geeignetes Instrument zur Selbstbeurteilung von Schmerzen dar. Da bei Patienten mit mäßiger bis starker kognitiver Beeinträchtigung keine der untersuchten Methoden der Selbstbeurteilung genügend anwendbar ist, erfordern diese eine Fremdbeurteilung. Weitere Forschung sollte die verschiedenen Instrumente zur Fremdbeurteilung vergleichen.

\section{Literatur}

1 Abbey J, Piller N, de Bellis A et al. The Abbey Pain Scale: a 1-minute numerical indicator for people with end stage dementia. International Journal of Palliative Nursing 2004; 10: 6-14

2 Carr ECJ, Mann EM. Schmerz und Schmerzmanagement. Praxishandbuch für Pflegeberufe. Bern: Huber; 2010

3 Closs SJ, Barr B, Briggs $M$ et al. A Comparison of Five Pain Assessment Scales for Nursing Home Residents with Varying Degrees of Cognitive Impairment. Journal of Pain and Symptom Management 2004; 27: $196-205$

4 Deutsches Netzwerk für Qualitätsentwicklung in der Pflege (DNQP) Expertenstandard Schmerzmanagement in der Pflege. Osnabrück: DNQP; 2005

5 Ferrell BA, Ferrell BR, Rivera L. Pain in cognitively impaired nursing home patients. Journal of Pain and Symptom Management 1995; 10: $591-598$

6 Folstein MF, Folstein SE, McHugh PR. "Mini-mental state": A practical method for grading the cognitive state of patients for the clinician. Journal of Psychiatric Research 1975; 12: 189-198

7 Fuchs-Lacelle S, Hadjistavropoulos HD. Development and preliminary validation of the Pain Assessment Checklist for Seniors with Limited Ability to Communicate (PACSLAC). Pain Management Nursing 2004; 1: $37-49$

8 Hadjistavropoulos T, Herr K, Turk DC et al. An Interdisciplinary Expert Consensus Statement on Assessment of Pain in Older Persons. Clin J Pain 2007; 23 (Suppl 1): 1-43

9 Helme RD, Gibson SJ. Measurement and Management of Pain in Older People. Australian Journal on Ageing 1998; 17: 5 -9

10 Horgas AL, Elliott AF, Marsiske M. Pain assessment in persons with dementia: relationship between self-report and behavioral observation. Journal of the American Geriatrics Society 2009; 57: 126-132

11 International Association for the Study of Pain (IASP). The Faces Pain Scale - Revised; 2001, www.iasp-pain.org/AM/Template.cfm?Section= Download_the_FPS_R\&Template=/CM/ContentDisplay.cfm\&Conten$\mathrm{tID}=14255$ (05.04.2013)

12 Knipping C. Lehrbuch Palliative Care. Bern: Huber; 2006

13 Lefebvre-Chapiro S. The Doloplus-2 scale - Evaluating pain in the elderly. European Journal of Palliative Care 2001; 8: 191 - 194

14 Lucas NP, Macaskill P, Irwig L et al. The development of a quality appraisal tool for studies of diagnostic reliability (QAREL). Journal of Clinical Epidemiology 2010; 63: 854-861

15 Morrison RS, Siu AL. A comparison of pain and its treatment in advanced dementia and cognitively intact patients with hip fracture. J Pain Symptom Manage 2000; 19: 240 - 248

$16 \mathrm{Ni}$ Thuathail A, Welford C. Pain assessment tools for older people with cognitive impairment. Nursing Standard 2011; 26: 39-46

17 Oesch P, Hilfiker R, Keller $S$ et al. Assessments in der Rehabilitation. Bd. 2: Bewegungsapparat. Bern: Huber; 2011

18 Pautex S, Herrmann F, Le Lous P et al. Feasibility and Reliability of Four Pain Self-Assessment Scales and Correlation With an Observational Rating Scale in Hospitalized Elderly Demented Patients. Journal of Gerontology 2005; 60A: 524-529

19 Pautex S, Michon A, Guedira $M$ et al. Pain in Severe Dementia: Self-Assessment or Observational Scales? Journal of the American Geriatrics Society 2006; 54: $1040-1045$

20 Pesonen A, Kauppila T, Tarkila $P$ et al. Evaluation of easily applicable pain measurement tools for the assessment of pain in demented patients. Acta Anaesthesiologica Scandinavica 2009; 53: 657-664

21 Royal College of Physicians and British Geriatrics Society \& British Pain Society (RCP). The assessment of pain in older people: national guidelines. Concise guidance to good practice series, No 8. London: RCP; 2007

22 Scherder EJA, Bouma A. Visual Analogue Scales for Pain Assessments in Alzheimer's Disease. Gerontology 2000; 46: 47-53

23 Schwermann M, Münch M. Professionelles Schmerzassessment bei Menschen mit Demenz. Ein Leitfaden für die Pflegepraxis. Stuttgart: Kohlhammer; 2008

24 Stolee P, Hillier LM, Esbaugh J et al. Instruments for the Assessment of Pain in Older Persons with Cognitive Impairment. Journal of the American Geriatrics Society 2005; 53: 319-326

25 Ware LJ, Epps CD, Herr K et al. Evaluation of the Revised Faces Pain Scale, Verbal Descriptor Scale, Numeric Rating Scale and Iowa Pain Thermometer in Older Minority Adults. Pain Management Nursing 2006; 7: $117-125$ 